\title{
Effect Zirconia's Phases in the Production of Hydroxyapatite
}

Kabir A. Jurado Villalobos, Armando Reyes Rojas, María del Carmen Aragón Duarte, Hilda E. Esparza-Ponce ${ }^{*}$

Departamento de Física, Centro de Investigación en Materiales Avanzados S.C. (CIMAV), Miguel de Cervantes 120, Complejo Industrial, C.P. 31109, Chihuahua, México.

The different tested biomaterials as potential bone grafts which include: ceramics, polymers, metals, composites and hybrid materials. The low bonding strength of plasma sprayed hydroxyapatite (HA) coating has been a point of potential weakness in its application as biomedical prosthesis. There is a continuous interest on controlling the mechanical properties [1]. Zirconia is a good biomaterial, has good mechanical properties and can be used as coating on alloys and titanium for production of apatite.

The present work was focused on the evaluation of addition effect of monoclinic, cubic and zirconia partial stabilized phases as thin film in the formation of apatite. The Thin films were deposited by sputtering RF on titanium and stainless steel substrates. The surface of thin films was immersed in Simulated Body Fluid (SBF) The SBF was prepared mixing sodium chloride, sodium bicarbonate, potassium chloride, calcium chloride, dibasic potassium phosphate, and magnesium chloride in deionized water [2-3].

The microstructural analyses of the samples were carried out by AFM and SEM. The crystalline state and the identification of phases were verified by X-ray diffraction (XRD) analysis using a diffractometer PAnalytical. Diffraction patterns were acquired from 20 to $100^{\circ} 2 \theta$ using a Scanning speed of $0.2 \% \mathrm{~min}$ with $\mathrm{K} \alpha \mathrm{Cu}$ radiation and grazing incidence mode. Figure 1 show the typical growth of cubic thin films on stainless steel and titanium. Figure No.2 shows the thin film morphology by AFM a) monoclinic ,c) PSZ and e)cubic phase onto stainless steel and figure 2 b) monoclinic, d) PSZ and f) cubic phase on Titanium. The XRD data indicates the crystallization of apatite on all phases which was identified by the JCPDS card as 00-086-1201.Figure 3 and 4 shown the morphology of hydroxyapatite by SEM on thin films with monoclinic, PSZ and cubic phase onto stainless steel and Titanium after one and 21 days of immersion, respectively. Finally, although in all phases hydroxyapatite is formed in monoclinic phase has a further development and preferably on titanium substrates.

References:

[1] K.A. Khora,*, L. Fua, V.J.P. Lima, P. Cheang Materials Science and Engineering A276 (2000) $160-166$.

[2] Kokubo T, Takadama H. Biomaterials. 2006 May;27(15):2907-15. Epub 2006 Jan 31. [3] A. B. Martínez-Valencia, G. Carbajal-De la Torre, A. Duarte Moller, H. E. Esparza-Ponce and M. A. Espinosa-Medina, International Journal of the Physical Sciences Vol. 6(29), pp. 66816691, 16 November, 2011.

The authors acknowledge funding from the Mexican Government (CONACyT) through project No.24463 CB-2005-01-51478. 


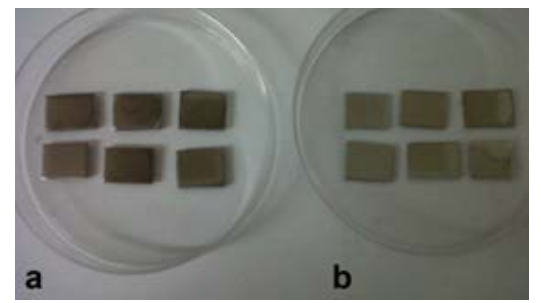

Figure 1. Thin films deposited by sputtering RF onto a) stainless steel and b) Titanium.

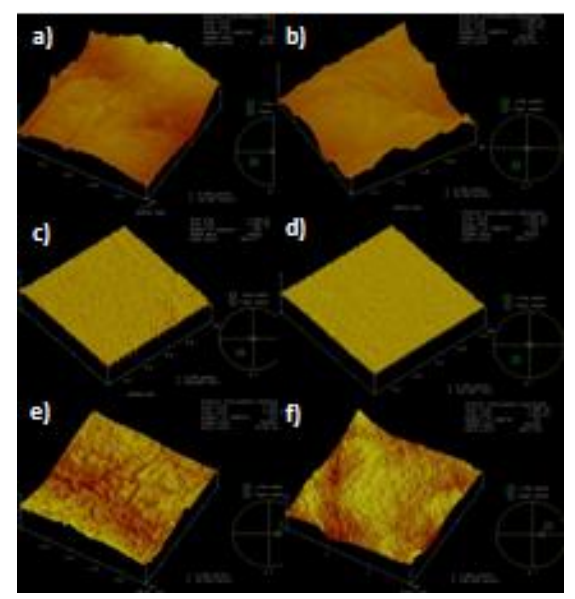

Figure 2. Morphology by AFM of thin films as deposited by sputtering RF a) monoclinic ,c)PSZ and e)cubic phase on stainless steel and b) monoclinic, d) PSZ and f) cubic phase on Titanium.

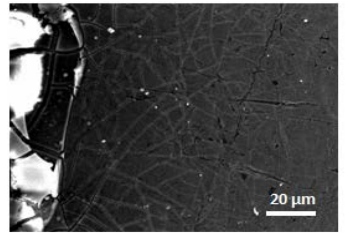

a)

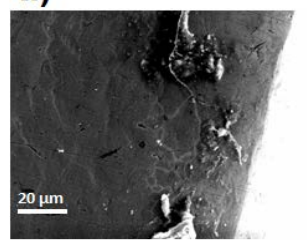

c)

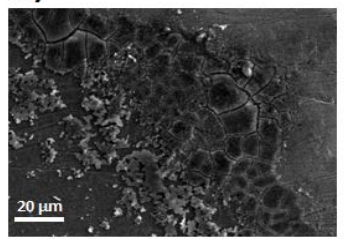

e)

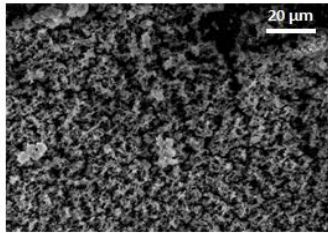

b)

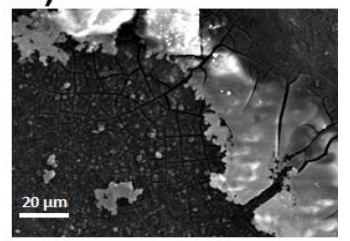

d)

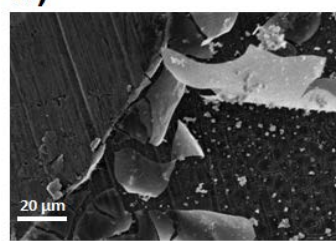

f)

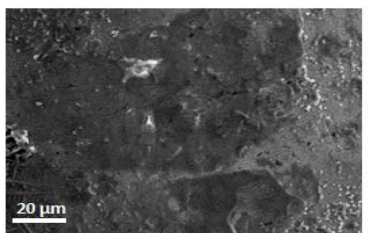

a)

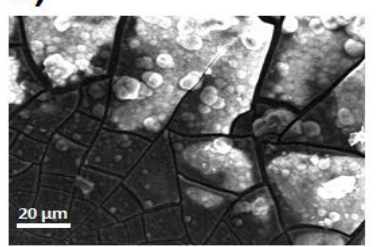

c)

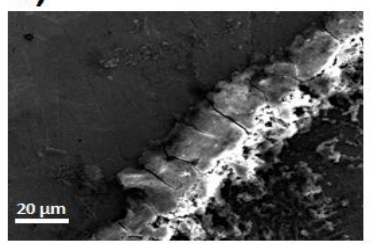

e)

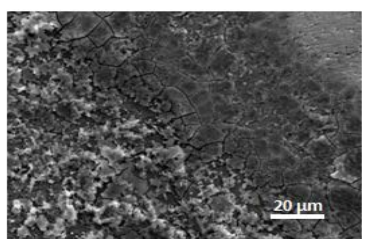

b)

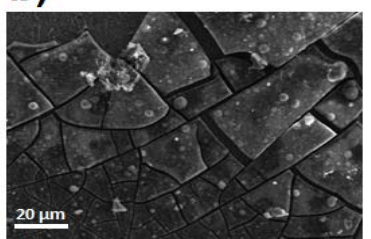

d)

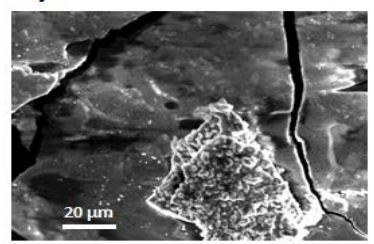

f)

Figure 3. Morphology of hydroxyapatite by SEM growth on thin films a) monoclinic, c) PSZ and e)cubic phase on stainless steel and b) monoclinic, d) PSZ and f) cubic phase on Titanium formed after one day of immersion.

Figure 4. Morphology of hydroxyapatite by SEM growth on thin films a) monoclinic, c) PSZ and e)cubic phase on stainless steel and b) monoclinic, d) PSZ and f) cubic phase on Titanium formed after 21 days of immersion. 